\title{
19 University
}

Wessmann, A., Volk, H.A., Packer, R.M.A., Ortega, M., and Anderson, T.J. (2016)

Quality-of-life aspects in idiopathic epilepsy in dogs. Veterinary Record, 179(9), 229. (doi:10.1136/vr.103355)

There may be differences between this version and the published version. You are advised to consult the publisher's version if you wish to cite from it.

http://eprints.gla.ac.uk/120278/

Deposited on: 20 September 2016

Enlighten - Research publications by members of the University of Glasgow http://eprints.gla.ac.uk 


\section{Print Summary}

2

\section{QUALITY OF LIFE ASPECTS IN IDIOPATHIC EPILEPSY IN DOGS}

4 A. Wessmann ${ }^{1,2}$, H.A. Volk ${ }^{3}$, R.M.A. Packer $^{3}$, M. Ortega ${ }^{2,4}$, T.J. Anderson ${ }^{2}$

5

$6 \quad{ }^{1}$ Pride Veterinary Centre, Derby, UK; ${ }^{2}$ University of Glasgow Veterinary School (UGVS),

7 Glasgow, UK; ${ }^{3}$ Royal Veterinary College (RVC), London, UK; ${ }^{4}$ Centro Clínico Veterinario 8 Indautxu, Bilbao, Spain;

9 


\section{ABSTRACT}

11 Quality of life (QoL) plays a significant role in the treatment of dogs with idiopathic epilepsy

12 (IE) yet is so far understudied. This study describes the outcome evaluation of an online questionnaire based on the carer's perception focusing on 62 QoL-questions in 159 dogs with IE.

Results showed that seizure frequency, but not seizure severity or presence of cluster seizures was significantly associated with carer perceived dog's QoL. Dogs receiving third line antiepileptic drugs had a significantly lower perceived QoL than those that did not. Generalised linear mixed model analysis demonstrated that severity of the side effects sleeping more and ataxia were significantly associated with carer perceived dog's QoL, with higher severities predicting lower QoL scores. The degree of carer acceptability of seizure

21 frequency and severity was significantly associated with the dog's reported seizure frequency and severity. Moreover, there was a significant association between IE-related QoL changes of the dog and the carer, with reductions in perceived canine QoL scores associated with reductions in carer QoL, and vice versa. In conclusion, aspects of canine IE can affect both the carer and their dog's QoL. This has implications for the management and requires consideration when treatment options and outcomes are discussed.

Keywords: Comorbidity, welfare, seizures, questionnaire 
33 Canine epilepsy studies mainly focus on the physical aspects of seizures, the impact of antiepileptic drugs (AED) and more recently also behavioural changes associated with the disease (Lord and Podell 1999, Chang and others 2006, Shihab and others 2011, Muñana and others 2012). The physical aspects of canine idiopathic epilepsy (IE) such as seizure frequency and severity as well as AED side effects are well described, yet the suspected impact on QoL has only been studied in small numbers of affected dogs. Carers of 37 dogs with IE were mostly concerned about the dog's QoL, adequate seizure frequency (less than one seizure every three months) and acceptable side effects of AEDs and that these factors would determine if seizure control was adequate (Chang and others 2006).

Neurobehavioral changes are a reported comorbidity of epilepsy in dogs and humans and include cognitive, behavioural or emotional changes associated with central nervous system dysfunction. These comorbidities in dogs include increased fear/anxiety, defensive aggression, abnormal perception, inattention, excitability/impulsivity, and show remarkable similarities to its human counterpart resembling anxiety and attention deficit hyperactivity disorder (Shihab and others 2011, Jokinen and others 2015, Packer and others 2016). The carer commonly assesses non-metric aspects like neurobehavioral changes and QoL aspects based on their perception (Lord and Podell 1999, Chang and others 2006, Shihab and others 2011, Muñana and others 2012, Packer and others 2016). The carer's perception of their dog's QoL may be biased but with this in mind the carer can serve as surrogate. This is an accepted practice not only in veterinary medicine but also in childhood epilepsy where usually the parent assesses the QoL of the epileptic child (Liu and Han 2015). It is further known that not only the QoL of the affected child but also of their carer can be affected in childhood epilepsy. Parents of epileptic children showed significantly lower QoL scores, and higher 
57 levels of depression and anxiety correlating to seizure control, employment, financial 58 implications of caring for an individual with epilepsy, the occurrence of status epilepticus, drug side effects and age of parents among others (Lv and others 2009). Information about the impact of caring for an epileptic dog on the carer's QoL is limited yet effect on the carer's day-to-day activities and their free time are describes (Lord and Podell 1999, Chang and others 2006).

63

Wessmann et al. (2014) published the creation of a disease-specific QoL questionnaire in dogs with IE and their carers (EpiQoL). The questionnaire design was based on the multidimensional aspects of QoL as defined by the WHO into physical, social, and neurobehavioral (dog) / psychological health aspects (carer) adapted for IE (Wessmann and others 2014). The current study explores the actual data and factors that affect QoL in dogs with IE and their carers with seven hypotheses (H1-H7) utilising data from Wessmann and others (2014). The QoL measures in this study largely focused on the carer's perception of their dog's and their own QoL.

H1: The seizure phenotype (frequency of seizure days, severity) affects the carer's perception of QoL in canine IE patients.

H2: There is an effect of being treated with certain AEDs upon carer perception of canine epilepsy.

H3: Side effects of AEDs impact upon carer perception of canine QoL.

H4: The reported severities of AED side effects are associated with the degree of carer acceptability of AED side effects.

H5: A dog's seizure frequency is associated with the degree of carer acceptability of seizure frequency. 
H6: A dog's seizure severity is associated with the degree of carer acceptability of seizure severity.

H7: There is an association between changes in carer QoL and carer perception of their dog's QoL following the onset of IE.

\section{MATERIAL AND METHODS}

Carers of dogs diagnosed with IE were recruited at the authors' institutions (UGVS, RVC) by paper mail stating a link to the online questionnaire, through contacting 800 primary care practices by email and though a canine epilepsy website (www.canineepilepsy.co.uk). Data was acquired from January to November 2011. Dogs were included if they presented with recurrent seizures (two or more) at least one month apart which were either presumptively diagnosed with IE following substantial investigation, including normal brain imaging (MRI or CT) and cerebrospinal fluid analysis independent of the age of onset or strongly suspected to have IE with seizures for more than one year and an age of onset between 6 months to 6 years. Responses were excluded if other diseases that required ongoing veterinary treatment or attention were reported or if the dog was not alive at the time of completion of the questionnaire (Wessmann and others 2014).

This study reports the outcome of 36 key questions (EpiQoL) associated with IE in 159 affected dogs and their carer's, as previously described by the authors (Supplementary file A, Wessmann and others 2014). Items such as seizure severity, frequency and side effects of AEDs were considered to affect the physical aspects of the dog. The seizure frequency considered the 'seizure days' on which seizures occurred and therefore could include single and multiple seizures ('cluster') for this day. Items such as restrictions and frustrations on the carer's life such as limitations in work, education, day-to-day activities, and social life because of caring for an epileptic dog were grouped under the social aspects affecting the 
carer. Psychological health aspects affecting the carer included items such as the carer's

108 distaste of AED side effects and carer anxiety around the seizure frequency and severity and

109 its effects on the dog (Supplementary file A). Twenty-six additional questionnaire items from

110 the original project questionnaire were included such as direct QoL focused questions,

111 descriptive data around the seizure event, psychological health aspects of the carer concerning

112 mainly the dog's health and the effect on the carer (Supplementary file B). A total of 62

113 questions were used to test the aforementioned hypotheses.

\section{Statistical analysis}

116 To investigate the above seven questions, three carer-reported proxies of canine QoL were

117 used as outcome measures:

118 (i) A score from 1-10 (treated as continuous, with 1 being the worst and 10 being the best);

119 (ii) QoL in the past three months (categorical from pretty bad-very well); and

120 (iii) Change in QoL since the onset of epilepsy (categorical from much decreased-much

121 increased).

122 Independent dog-related variables included aspects of the seizure phenotype: seizure

123 frequency (seizure days), seizure severity and the occurrence of cluster seizures; AED

124 treatment and the severity of side effects encountered. Carer-related independent variables

125 included the degree of carer acceptability (rated from strong agreement - strong

126 disagreement) of seizure frequency and severity, and carer reported QoL change since the

127 onset of epilepsy (categorical from much decreased-much increased). Kruskal-Wallis and

128 Mann-Whitney U tests were used to test for associations between outcome measure (i) and

129 independent variables, and Chi-squared analysis for associations between outcome measures

130 (ii) and (iii) and independent variables. Where indicated by univariate analyses $(\mathrm{p}<0.10)$,

131 generalised linear mixed model (glmm) analyses were carried out, with breed taken into 
account as a random effect. Multicollinearity was checked for in all models, identified from

133 inflated standard errors in the models, and thus avoided. Model fit was assessed using the

134 deviance and Akaike's information criterion. All tests were used two-sided with $\mathrm{P}<0.050$

135 being considered statistically significant.

\section{RESULTS}

138 One hundred-and-fifty-nine dogs of 50 breeds met the inclusion criteria. Mean age was 5.8 139 years (median 5.2 years, range 0.7-12.5 years), with 66 female (52 neutered) and 93 male 140 dogs (67 neutered). The mean age of onset of seizures was 2.7 years (median 2 years, range $141 \quad 0.3-9.0$ years $).$

143 The results of the 36 key questions and the additional 26 questionnaire items are displayed in 144 Supplementary file A and B respectively. The answers to the seven carer perceived QoL 145 questions are as follows:

147 H1: The seizure phenotype (frequency of seizure days, severity) affects the carer's perception 148 of QoL in canine IE patients.

149 Of the three measures of seizure phenotype (seizure frequency, severity and presence of 150 cluster seizures), only one measure, seizure frequency, was significantly associated with carer 151 perceived dog's QoL, when scored out of ten (Kruskal-Wallis=17.5, $\mathrm{p}=0.014$ ), when rated for 152 the past three months categorically $\left(\mathrm{X}^{2}=38.8, \mathrm{p}=0.003\right)$, and when questioned about how it 153 has changed since the onset of $\operatorname{IE}\left(\mathrm{X}^{2}=41.2, \mathrm{p}=0.016\right)$. Higher seizure frequencies were 154 associated with decreased carer perceived dog's QoL measures, with the median QoL score 155 for dogs experiencing less than one seizure day every six months scoring 9 (range 5-10), $15641.4 \%$ of carers perceived their dog's QoL as 'very well: could hardly be better' and $51.7 \%$ 
stating their dog's QoL had stayed the same since the onset of epilepsy. In contrast, dogs experiencing 'more than one seizure day every week' had a median QoL score of 7.5 (range

4-8), with no carers reporting their dog's QoL 'very well: could hardly be better' and the majority (87.5\%) stating their dog's QoL had 'decreased a little' since the onset of epilepsy. There were no associations between seizure severity and the presence of cluster seizures with any measure of QoL ( $\mathrm{p}>0.050)$.

\section{H2: There is an effect of being treated with certain AEDs upon carer perception of canine} epilepsy.

There was no difference in carer perceived dog's QoL scored out of ten or in the past three months between dogs receiving phenobarbital or not, potassium bromide or not, or diazepam or not $(\mathrm{p}>0.050)$. When QoL was considered since the onset of epilepsy, dogs that received phenobarbital were rated by their carers to have had a reduction in QoL compared to those that did not (55.6\% vs. $25.7 \%$ rated their dog's QoL to be 'a little decreased' since the onset of epilepsy, respectively; $\mathrm{p}=0.008$ ), as were dogs that received potassium bromide in comparison to those that did not $(60.5 \%$ 'a little decreased' vs. $36.8 \%$, respectively; $\mathrm{p}=0.017)$. When the number of AEDs administered was considered, there was a significant difference in carer perceived dog's QoL between dogs being treated with third line drugs or not, when scored out of ten (Mann-Whitney=1545, p=0.002), rated for the past 3 months categorically $\left(X^{2}=10.8 \mathrm{p}=0.013\right)$, and when questioned about how it has changed since the onset of IE $\left(X^{2}=\right.$ 13.5, $\mathrm{p}=0.009$ ), with dogs receiving third line drugs having a reduced QoL compared to those that do not.

\section{H3: Side effects of AEDs impact upon carer perception of canine QoL (Table 1)}


181 The severity of four of the eleven reported AED side effects were significantly associated

182 with carer perceived dog's QoL at the univariate level: 'drinking more' (Kruskal-Wallis $183(\mathrm{KW})$ : 15.5, $\mathrm{p}=0.008)$, 'sleeping more' $(\mathrm{KW}: 14.8, \mathrm{p}=0.011)$, 'wobbly/not coordinated when walking' (KW: 16.3, p=0.006) and 'restlessness/pacing' (KW: 21.0, p=0.001). Dogs that were not affected by 'drinking more' had a median carer perceived QoL score of 9.0 (range 7.0186 10.0), whereas those reported to be very severely affected had a median of 8.0 (range 3.0187 10.0). Dogs that were not affected by 'sleeping more' had a median carer perceived QoL score of 9.0 (range 5.0-10.0), whereas those reported to be very severely affected had a median of 8.0 (range 4.0-10.0). Dogs that were not affected by 'wobbliness/not coordinated 190 when walking' had a median carer perceived QoL score of 9.0 (range 6.0-10.0), whereas 191 those reported to be very severely affected had a median of 8.0 (range 5.0-10.0). Finally, dogs 192 that were not affected by 'restlessness/pacing' had a median carer perceived QoL score of 9.0 193 (range 3.0-10.0), whereas those reported to be very severely affected had a median of 8.0 194 (range 3.0-10.0). These four factors were tested in a generalised linear mixed model (glmm) 195 with breed taken into account as a random effect. While four factors were significantly 196 associated with QoL at the univariate level as stated above, only two factors remained 197 significant when included together in a glmm showing the largest effect on QoL that was not 198 explained by the other variables. These two factors significantly predicted carer perceived 199 dog's QoL: the severity of 'sleeping more' and the severity of being 'wobbly/not coordinated 200 when walking' $(\mathrm{p}<0.050)$. The less severely the dog was affected by 'sleeping more' or being 201 'wobbly/not coordinated', the higher (better) the carer perceived dog's QoL score. acceptability of AED side effects. 
The severity of seven of the eleven reported AED side effects (rated from 1-5: very mild -

206 very severe) were significantly associated with carer reported acceptability of side effects 207 (rated from 1-5: strongly agree - strongly disagree) at the univariate level: 'eating more', 208 'gaining weight', 'drinking more', 'urinating more', 'sleeping more', 'wobbly/not coordinated 209 when walking' and 'restlessness/pacing' ( $\mathrm{p}<0.050)$. Increased severity of these side effects 210 was associated with a decreased level of carer acceptability. There was no association 211 between the severity of 'itchiness/skin rash', 'vomiting', 'diarrhoea' and 'coughing' and carer-rated acceptability of side effects $(\mathrm{p}>0.050)$.

H5: A dog's seizure frequency is associated with the degree of carer acceptability of seizure 215 frequency.

216 Seizure frequency was significantly associated with the degree of carer acceptability of 217 seizure frequency $\left(X^{2}=100.5, \mathrm{p}<0.001\right)$, with carers reporting higher seizure frequencies 218 disagreeing more that their dogs seizure frequency was acceptable and vice versa. For 219 example, $46.4 \%$ of the carers of dogs experiencing 'less than one seizure day every six 220 months' strongly agreed their dog's seizure frequency was acceptable, whereas no carers of 221 dogs experiencing 'more than one seizure day every week' strongly agreed their dog's seizure 222 frequency was acceptable, with $75.0 \%$ strongly disagreeing.

224 H6: A dog's seizure severity is associated with the degree of carer acceptability of seizure 225 severity.

226 Seizure severity was significantly associated with the degree of carer acceptability of seizure 227 severity $\left(X^{2}=100.9, \mathrm{p}<0.001\right)$, with carers reporting higher seizure severities disagreeing more 228 that their dog's seizure severity was acceptable and vice versa. For example, $37.5 \%$ of the 229 carers of dogs experiencing 'mild' seizures strongly agreed their dog's seizure severity was 
acceptable, whereas only $4.8 \%$ of carers of dogs experiencing 'very severe' seizures strongly

231 agreed, with $61.9 \%$ strongly disagreeing that this severity was acceptable.

H7: There is an association between changes in carer QoL and carer perception of their

235 There was a significant association between the change in carer perceived dog's QoL after the 236 onset of IE and the change in the carer's QoL after the onset of IE $\left(X^{2}=101.7, p<0.001\right)$, with carers reporting their dog's QoL had decreased more likely to report that their QoL had decreased too, and vice versa. No carers of dogs whose perceived QoL had 'much decreased' after the onset of epilepsy reported their own QoL was 'increased' or 'much increased' $240(0.0 \%)$, with $50.0 \%$ stating their QoL was also 'much decreased'. Participants commented on 241 suffering to some degree from depression or panic attacks $(29.0 \%)$ and feeling isolated $242(22.0 \%)$ as a result of caring for an epileptic dog (Supplementary file B). In contrast, the majority $(71.4 \%)$ of carers who perceived their dog's QoL was 'much increased' after the onset of epilepsy reported their own QoL was also 'much increased'.

\section{DISCUSSION}

247 Of the three measures of seizure phenotype (seizure frequency, severity and presence of 248 cluster seizures), only one measure, seizure frequency (seizure days), was significantly 249 associated with QoL as perceived by the dog's carer in this study. Chang and others (2006) 250 reported that carers of 29 dogs referred to one institution perceived a seizure frequency of 251 'one seizure every three to six months' to be most reasonable for their pet. Most participants 252 in the current study perceived only a 'seizure-free' state acceptable for their pet 253 (Supplementary file B). Similarly, freedom from seizures is the treatment goal in people (Lee 254 2014). It was shown that seizure frequency is one of the main risk factor for decreased QoL in 
children with epilepsy (Liu and Han 2015). A significant correlation between seizure severity

256 and the carer's perception of their dog's QoL could not be established. The statistical analysis 257 did not show whether a history of 'cluster seizures' was associated with the dog's QoL. It 258 appears that the frequency but not their temporal density was important to carer perceived 259 QoL of their dog. It would appear reasonable to assume that cluster seizures and status 260 epilepticus impact on the perceived dog's QoL, given that the occurrence of cluster seizures 261 and status epilepticus increased the risk of epilepsy related euthanasia in previous studies 262 (Saito and others 2001, Monteiro and others 2012, Fredso and others 2014). This might be related to the fact that the QoL scores are a reflection of the carer's perception of their dog's QoL as a proxy of the dog's actual QoL.

The use of two common types of AED (phenobarbital and potassium bromide) had a negative effect on carer's perception of their dog's QoL when considered since the onset of epilepsy, and there was an effect of the number of AEDs administered, with dogs being treated with third line drugs experiencing a reduced carer perceived QoL compared to those with 1-2 AEDs. Both, seizure control and number of medications administered, are significantly associated with QoL in epileptic children (Williams and others 2003). Drug-resistance is frustrating and challenging to manage. The probability of seizure control is reduced with successive AED treatment (Lee 2014, Packer and others 2015). Response rates in people with epilepsy for the first, second or third-line AED as proportion of the population were 47-50\%, 10-13\% and 2-4\% respectively (Kwan and Brodie 2000, Mohanraj and Brodie 2006). 276 Similarly, in dogs with epilepsy, the response rate as proportion of the population for first, 277 second and third line AEDs was 37\%, 11\% and 6\% respectively (Packer and others 2015). 278 There was an association between both seizure frequency and severity and carer-perceived 279 acceptability of these traits, with higher seizure frequencies and severities perceived to be less 
acceptable by carers. Drug-resistance remains a main cause of epilepsy related euthanasia in

281 canine IE (Chang and others 2006, Fredso and others 2014, Wessmann and others 2014).

282 Although freedom from seizures is one of the main goals of epilepsy therapy in people (Lee 2014), it is not easily achieved, and with as few as $14 \%$ of treated dogs achieving remission in hospital dog populations (Packer and others 2014) the management of carer's expectations by their veterinarians is vital for understanding the outcomes of therapy.

The AED side effects that impact on the dog's QoL and their acceptability by the carer vary between the 11 investigated side effects. Only the AED side effects 'sleeping more' and the severity of being 'wobbly/not coordinated when walking' significantly predicted carer perception of their dog's QoL in a multivariate analysis. 'Drinking more' and 'restlessness/pacing' had further a significant influence on the dog's QoL at a univariate level. However, increased severity of these side effects 'eating more', 'gaining weight', 'drinking more', 'urinating more', 'sleeping more', 'wobbly/not coordinated when walking' and 'restlessness/pacing' were associated with a decreased level of carer acceptability. The variety of the different side effects may explain discrepancies to previous studies. One study reported that phenobarbital therapy appeared to have minimal side effects on the overall carer perceived QoL of the studied dog population and thus did not produce a significant problem for the carers (Lord and Podell 1999), whereas another study reported that acceptable side effects were one of the greatest concerns for carers (Chang and others 2006). The presence of 300 side effects is an outcome measure for successful AED therapy in people (Lee 2014). Newer 301 AEDs commonly fail to show better efficacy than older AEDs. Thus, the selection of the first302 line drug is mostly lead by the characteristics and frequency of the AED side effects (Lee 2014). With the advent of newer AEDs in veterinary medicine AED side effects have the potential to drive drug selection, given the perceived impact of the side effects on the QoL of 
the dog by the carer. Despite multiple AEDs being available in human medicine, there

306 remains a need for new AEDs in canine and human epilepsy with fewer side effects, increased

307 efficacy, drugs with different mechanism of action with the potential of synergistic

308 combination therapy (Lee 2014). On the other hand, third AED currently used in veterinary

309 medicine have largely not been through clinical trials in dogs to test their efficacy. Therefore

310 one could question the necessity of finding even newer if the available ones have not been

311 tested.

313 The change in the carer's perception of their dog's QoL was significantly associated with the

314 change in the carer's QoL after the onset of IE. Carers reporting a decreased QoL in their 315 dogs were more likely to report that their QoL had also decreased. This response reflects a 316 well-known phenomenon in childhood epilepsy, where the disease not only affects the QoL of 317 the affected child but also of the carer, usually their parent (Cushner-Weinstein and others 318 2008, Lv and others 2009). The factors correlated with parental QoL were seizure control, 319 status epilepticus, drug side effects, the degree of the child's anxiety and depression (Lv and 320 others 2009). Lack of control over events, unpredictability of events, sleep deprivation and a 321 feeling of helplessness are known factors in the development of stress in people (Henn and 322 Vollmayr 2005, Koolhaas and others 2011) and epilepsy influences some, if not all of these 323 factors. Moreover, up to $50 \%$ of mothers are at risk of clinical depression as a consequence 324 caring for an epileptic child (Ferro and Speechley 2009). Similarly, canine IE can also impact 325 on the mental health of the carer. A small number of participants commented on suffering to 326 some degree from depression or panic attacks and feeling isolated as a result of caring for an 327 epileptic dog (Supplementary file B). Noteworthy is that an improved carer perceived dog's 328 QoL resulted also in an improved QoL of the carer demonstrating potentially positive aspects 329 of IE treatment. This positive finding associated with IE is encouraging and may reflect an 
330 enhanced connection between diseased pet and carer observed for cats with diabetes mellitus

331 and their carers (Niessen and others 2010).

333 This study showed on a large scale, that canine IE has not only an effect on the perceived QoL

334 of the affected dog but is also significantly associated with the carer's perceived QoL. Carers 335 reporting a decreased QoL in their dogs were more likely to report that their QoL had 336 decreased too. Seizure frequency, severity of AED side effects sleeping more and ataxia and 337 dogs receiving third line AEDs were associated with the carer perceived dog's QoL, with 338 higher severities predicting lower QoL scores. Thus, optimising seizure control and AED 339 therapy will not only affect the perceived QoL of the affected dog but also of the carer. The 340 carer's QoL affected by caring for an epileptic dog is an important part of IE treatment as the 341 perceived impact of IE not only on the dogs' but also on the carers' QoL is likely to influence 342 a carer's choices regarding treatment or euthanasia and requires consideration when treatment 343 options are discussed.

\section{ACKNOWLEDGEMENTS}

346 The authors thank all carers, referring veterinary surgeons and www.canineepilepsy.co.uk for 347 their participation in the study. 


\section{REFERENCES}

351 1. BAKER GA, SMITH DF, DEWEY M, JACOBY A \& CHADWICK DW. (1993) The initial development of a health-related quality of life model as an outcome measure in epilepsy. Epilepsy Research 16, 65-81

2. CHANG, Y., MELLOR, D.J., \& ANDERSON, T.J. (2006) Idiopathic epilepsy in dogs: owners' perspectives on management with phenobarbitone and/or potassium bromide. Journal of Small Animal Practice 47, 574-581

3. CRAMER, J.A. for the ILAE Subcommission on Outcome Measurement in Epilepsy (CAMFIELD, C., CARPAY, H., HELMSTAEDTER, C., LANGFITT, J., MALMGREN, K. \&WIEBE, S.) (2002) Principles of health-related quality of life: assessment in clinical trials. Epilepsia 43, 1084-1095

4. CUSHNER-WEINSTEIN, S., DASSOULAS, K., SALPEKAR, J.A., HENDERSON, 362 S.E., PEARL, P.L., GAILLARD, W.D. \& WEINSTEIN, S.L. (2008) Parenting stress and childhood epilepsy: the impact of depression, learning, and seizure-related factors. Epilepsy \& Behavior 13, 109-114

5. ENGLOT, D.J., YANG, L., HAMID, H., DANIELSON, N., BAI, X., MARFEO, A., YU, L., GORDON, A., PURCARO, M.J., MOTELOW, J.E., AGARWAL, R., ELLENS, D.J., GOLOMB, J.D., SHAMY, M.C., ZHANG, H., CARLSON, C., DOYLE, W., DEVINSKY, O., VIVES, K., SPENCER, D.D., SPENCER, S.S., SCHEVON, C., ZAVERI, H.P. \& BLUMENFELD, H. (2010) Impaired consciousness in temporal lobe seizures: role of cortical slow activity. Brain 133, 3764-3777

6. FERRO, M.A. \& SPEECHLEY, K.N. (2009) Depressive symptoms among mothers of children with epilepsy: a review of prevalence, associated factors, and impact on children. Epilepsia 50, 2344-2354

7. FREDS $\emptyset$, N., KOCH, B.C., TOFT, N. \& BERENDT, M. (2014) Risk factors for survival 
in a university hospital population of dogs with epilepsy. Journal of Veterinary Internal Medicine 28, 1782-1788

8. HENN, F.A. \& VOLLMAYR, B. (2005) Stress models of depression: forming genetically vulnerable strains. Neuroscience and Biobehavioral Reviews 29, 799-804

9. JOKINEN, T.S., TIIRA, K., METSÄHONKALA, L., SEPPÄLÄ, E.H., HIELMBJÖRKMAN, A., LOHI, H., \& LAITINEN-VAPAAVUORI, O. (2015) Behavioral Abnormalities in Lagotto Romagnolo Dogs with a History of Benign Familial Juvenile Epilepsy: A Long-Term Follow-Up Study. Journal of Veterinary Journal of Medicine May 6. doi: 10.1111/jvim.12611 [Epub ahead of print]

10. KASPER, B.S., KASPER, E.M., PAULI, E. \& STEFAN, H. (2010) Phenomenology of hallucinations, illusions, and delusions as part of seizure semiology. Epilepsy \& Behavior 18, $13-23$

11. KOOLHAAS, J.M., BARTOLOMUCCI, A., BUWALDA, B., DE BOER, S.F., FLÜGGE, G., KORTE, S.M., MEERLO, P., MURISON, R., OLIVIER, B., PALANZA, P., RICHTER-LEVIN, G., SGOIFO, A., STEIMER, T., STIEDL, O., VAN DIJK, G., WÖHR, M. \& FUCHS, E. (2011) Stress revisited: a critical evaluation of the stress concept. Neuroscience and Biobehavioral Reviews 35, 1291-1301

12. KWAN, P. \& BRODIE, M. J. (2000) Early Identification of Refractory Epilepsy. New England Journal of Medicine 342, 314-319

13. LEE, S.K. (2014) Old versus New: Why Do We Need New Antiepileptic Drugs? Journal of Epilepsy Research 4, 39-44

14. LIU, X. \& HAN, Q. (2015) Risk Factors on Health-Related Quality of Life in Children With Epilepsy. Clinical Pediatrics 54 (14), 1334-1338

15. LORD, L.K. \& PODELL, M. (1999) Owner perception of the care of long-term phenobarbital-treated epileptic dogs. Journal of Small Animal Practice 40, 11-15 
16. LV, R., WU, L., JIN, L., LU, Q., WANG, M., QU, Y. \& LIU, H. (2009) Depression, anxiety and quality of life in parents of children with epilepsy. Acta Neurologica Scandinavica 120, 335-341

17. MOHANRAJ, R. \& BRODIE, M. (2006) Diagnosing refractory epilepsy: response to sequential treatment schedules. European Journal of Neurology 13, 277-282

18. MONTEIRO, R., ADAMS, V., KEYS, D. \& PLATT, S.R. (2012) Canine idiopathic epilepsy: prevalence, risk factors and outcome associated with cluster seizures and status epilepticus. Journal of Small Animal Practice 53, 526-530

19. MOREIRA, H., CARONA, C., SILVA, N., FRONTINI, R., BUllingER, M. \& CANAVARRO, M.C. (2013) Psychological and quality of life outcomes in pediatric populations: a parent-child perspective. The Journal of Pediatrics 163, 1471-1478

20. MUNAANA, K.R., THOMAS, W.B., INZANA, K.D., NETTIFEE-OSBORNE, J.A., MCLUCAS, K.J., OLBY, N.J., MARIANI, C.J. \& EARLY, P.J. (2012) Evaluation of levetiracetam as adjunctive treatment for refractory canine epilepsy: a randomized, placebo-controlled, crossover trial. Journal of Veterinary Internal Medicine 26, 341-348

21. NIESSEN, S.J., POWNEY, S., GUITIAN, J., NIESSEN, A.P., PION, P.D., SHAW, J.A. \& CHURCH, D.B. (2010) Evaluation of a quality-of-life tool for cats with diabetes mellitus. Journal of Veterinary Internal Medicine 24, 1098-1105

22. PACKER, R.M., LAW T.H., DAVIES, E., ZANGHI, B., PAN, Y., VOLK, H.A. (2016) Effects of a ketogenic diet on ADHD-like behavior in dogs with idiopathic epilepsy. Epilepsy \& Behavior 55, 62-68

23. PACKER, R.M., SHIHAB, N.K., TORRES, B.B. \& VOLK, H.A. (2014) Clinical risk factors associated with anti-epileptic drug responsiveness in canine epilepsy. PLoS One $\mathbf{9}$, e106026

24. PACKER, R.M., SHIHAB, N.K., TORRES, B.B., \& VOLK, H.A. (2015) Responses to 

successive anti-epileptic drugs in canine idiopathic epilepsy. Veterinary Record 176, 203

25. SAITO, M., MUÑANA, K.R., SHARP, N.J. \& OLBY, N.J. (2001) Risk factors for development of status epilepticus in dogs with idiopathic epilepsy and effects of status epilepticus on outcome and survival time: 32 cases (1990-1996). Journal of the American Veterinary Medical Association 219, 618-623

26. SHIHAB, N., BOWEN, J. \& VOLK, H.A. (2011) Behavioral changes in dogs associated with the development of idiopathic epilepsy. Epilepsy \& Behavior 21, 160-167

27. WESSMANN, A., VOLK, H.A., PARKIN, T., ORTEGA, M. \& ANDERSON, T.J. (2014) Evaluation of quality of life in dogs with idiopathic epilepsy. Journal of Veterinary Internal Medicine 28, 510-514

28. WILliAMS, J., STEEL, C., SHARP, G.B., DELOSREYES, E., PHILliPS, T., BATES, S., LANGE, B. \& GRIEBEL, M.L. (2003) Parental anxiety and quality of life in children with epilepsy. Epilepsy \& Behavior 4, 483-486 


\section{LEGENDS}

442

443

Table 1. Impact of antiepileptic drug side effects upon carer perceived dog's quality of

444 life score. The factors 'drinking more', 'sleeping more', 'wobbly/not coordinated when 445 walking' and 'restlessness/pacing' were tested in a generalised linear mixed model (glmm) 446 with breed taken into account as a random effect. The severity of 'sleeping more' and the 447 severity of being 'wobbly/not coordinated when walking' $(\mathrm{p}<0.050)$ significantly predicted 448 carer perceived dog's QoL. The less severely the dog is affected by 'sleeping more' or being 449 'wobbly/not coordinated when walking', the higher the carer perceived dog's QoL score.

451 Supplementary file A. Outcome of 7 themes with 36 key questions from a disease-specific 452 IE online questionnaire as previously published by Wessmann and others (2014).

453

454 Supplementary file B. Outcome of 26 complementary questionnaire items as previously 455 published by Wessmann and others (2014).

456

457

458 
460 life score. The factors 'drinking more', 'sleeping more', 'wobbly/not coordinated when walking' and 'restlessness/pacing' were tested in a generalised linear mixed model (glmm) with breed taken into account as a random effect. The severity of 'sleeping more' and the severity of being 'wobbly/not coordinated when walking' $(p<0.050)$ significantly predicted carer perceived dog's QoL. The less severely the dog is affected by 'sleeping more' or being 'wobbly/not coordinated when walking', the higher the carer perceived dog's QoL score.

\begin{tabular}{|c|c|c|c|c|c|}
\hline Risk factor & Sub-category & $\begin{array}{c}\text { Coefficient }(95 \% \\
\text { CI })\end{array}$ & SE & $\mathbf{t}$ & P value \\
\hline Intercept & - & $5.7(4.5-6.9)$ & 0.6 & 9.2 & 0.000 \\
\hline \multirow[t]{6}{*}{ Sleeping more } & Side effect not present & $1.7(0.4-2.9)$ & 0.6 & 2.6 & 0.010 \\
\hline & Very mild & $1.3(-0.1-2.6)$ & 0.7 & 1.8 & 0.072 \\
\hline & Mild & $1.5(0.2=2.9)$ & 0.7 & 2.2 & 0.030 \\
\hline & Moderate & $1.0(-0.3-2.3)$ & 0.6 & 1.96 & 0.114 \\
\hline & Severe & $1.4(-0.1-2.8)$ & 0.8 & 1.8 & 0.702 \\
\hline & Very severe & \multicolumn{4}{|c|}{ Reference } \\
\hline \multirow{6}{*}{$\begin{array}{l}\text { Wobbly/not } \\
\text { coordinated } \\
\text { when walking }\end{array}$} & Side effect not present & $1.4(0.4-2.4)$ & 0.5 & 2.8 & 0.006 \\
\hline & Very mild & $1.1(-0.0-2.1)$ & 0.5 & 2.0 & 0.053 \\
\hline & Mild & $0.7(-0.5-1.8)$ & 0.6 & 1.1 & 0.258 \\
\hline & Moderate & $0.3(-0.8-1.4)$ & 0.6 & 0.6 & 0.553 \\
\hline & Severe & $0.9(-0.3-2.1)$ & 0.6 & 1.5 & 0.138 \\
\hline & Very severe & \multicolumn{4}{|c|}{ Reference } \\
\hline
\end{tabular}

